\title{
Live Festival: Online streaming of student's moving image projects, in the age of COVID
}

\author{
Keywords \\ Covid-19, design, Live streaming, screening event, VFX
}

The Covid-19 pandemic has disrupted most social activities including education, and unexpectedly studying and working from home has become a new normality. However, this has presented challenges, including questions about how educators might curate 'end of the year exhibitions, presentations and screenings that have become an integral part of art school practice. These events constitute more than a 'celebratory finale' for art schools. They can also function as important learning environments. These types of events are usually formatted like film festivals. In the shadow of Covid-19, many film festivals around the world has gone online. Traditionally, Visual Effects (VFX) students at Auckland University of Technology (AUT), have showcased their VFX based short films, at the end of each semester in a popular event called Screening Night. This festival is not only a proud moment for students where they are able to showcase the outcome of their final to their friends, families and potential collaborators and employers, but it also supports networking processes. Every year, a panel of New Zealand based potential employers and peer artists are invited to these festivals and constructive dialogue occurs throughout and subsequent to the event. When Covid-19 occurred, Auckland city experienced an extensive lockdown, all social gatherings, including AUT's Screening Night were cancelled. This case study offers a pedagogical consideration of how an alternative method for screening events using live festivals via online steaming services might be designed and facilitated. Online live streaming has been a prominent medium for personal and individual broadcasters as diverse as video gamers, gadget reviewers, home chefs, and nurseries, through which they monetize content creation activities to create significant sources of revenue. Accordingly, such methods are familiar to students and propose themselves as potentials for educational application, design, and development. This case study demonstrates how the structure of a festival was migrated and reformatted for a live festival where students: 1- Introduced their work with a self-reflective speech, 2- Showcased their films and the vfx production breakdowns, 3- Received feedback and reviews from invited guests, 4- Received positive support from the curator, 5- Networked in a 'Pavilion space' and engaged in employability discussions. Then through a vigorous planning, the ethos of physical in-person screening night event has been translated into a live festival event. Students introduced their artwork in their online self-reflective speeches, guests were invited to comment and review artworks alongside the convener. Live festival event featured new functions including live interactive commenting. Also, many students were watching the live festival event with their families at their home, within their bubble so the spectrum out to the community seems wider. The study demonstrates that live steaming screening as a pedagogical method, may constitute an effective alternative to traditional, physical in-person screening events, not just in the era of Covid-19 restrictions but as an adaptable approach to future online festival, presentation, learning and networking initiatives. The recorded version of the first live festival at AUT can be viewed here: https://www.youtube.com/ watch?v=RyBGzovaunM 